\title{
ETS-1: A potential target of glycolysis for metabolic therapy by regulating glucose metabolism in pancreatic cancer
}

\author{
XIU ZHANG ${ }^{1 *}$, DAN WU $^{2 *}$, MOHANAD ALDAROUISH $^{1}$, XIAODONG YIN $^{3}$, \\ CHUNYAN $\mathrm{LI}^{4}$ and CAILIAN WANG ${ }^{1}$ \\ ${ }^{1}$ Department of Oncology, Zhongda Hospital, Medical School of Southeast University, Nanjing, Jiangsu 210009; \\ ${ }^{2}$ Department of Oncology, Jiangyin People's Hospital, Jiangyin, Jiangsu 224000; ${ }^{3}$ Department of Oncology, \\ Binhai People's Hospital, Yancheng, Jiangsu 224500; ${ }^{4}$ Nanjing Medical University, Nanjing, Jiangsu 210009, P.R. China
}

Received September 2, 2016; Accepted November 7, 2016

DOI: $10.3892 /$ ijo.2016.3770

\begin{abstract}
Pancreatic cancer is one of the most lethal malignancies of all types of cancer due to lack of early symptoms and its resistance to conventional therapy. In our previous study, we have shown that v-ets erythroblastosis virus E26 oncogene homolog-1 (ETS-1) promote cell migration and invasion in pancreatic cancer cells. However, the function of ETS-1 in regulation of glycolysis and autophagy during progression of pancreatic cancer has not been defined yet. In this study, we sought to identify the potential role for silencing ETS-1 in reducing the expression of glucose transporter-1 (GLUT-1) to disturb glycolysis through alteration of 'Warburg effect', by which could result in AMP-activated protein kinase (AMPK) activation, autophagy induction and reduction of cell viability. MTT assay was applied to assess the cell viability in ETS-1 silencing cells and control groups. Glucose absorption rate, lactate production rate and cellular ATP level were measured by standard colorimetric assay kits. The levels of mRNAs of ETS-1, GLUT-1, autophagy-related gene 5 (ATG5) and ATG7 were analyzed by qRT-PCR. The expression of ETS-1, GLUT-1, ATG5, ATG7, p-AMPK, and LC3II proteins were evaluated by western blot analysis. GraphPad Prism 5.0 was used for all statistical analysis. We found that cell viability was obviously attenuated after silencing ETS-1. Besides, our results also
\end{abstract}

Correspondence to: Professor Cailian Wang, Department of Oncology, Zhongda Hospital, Medical School of Southeast University, 87 Dingjiaqiao Road, Nanjing, Jiangsu 210009, P.R. China

E-mail: wangcailian65@hotmail.com

*Contributed equally

Abbreviations: ETS-1, v-ets erythroblastosis virus E26 oncogene homolog-1; GLUT-1, glucose transporter-1; AMPK, AMP-activated protein kinase; ATG5, autophagy-related gene 5; ATG7, autophagyrelated gene 7; GAPDH, glyceraldehyde-3-phosphate dehydrogenase; RT-PCR, reverse transcription-polymerase chain reaction

Key words: pancreatic cancer, ETS-1, GLUT-1, glycolysis, autophagy showed that the expression of GLUT-1 significantly declined in ETS-1 silencing cell lines which resulted in a lower glucose utilization and lactate production. Furthermore, the inhibition of glycolysis, which depends on glucose utilization and lactate production, reduced the generation of energy in the form of ATP. Moreover, the reduction of cellular ATP was associated with stimulation of AMP-activated protein kinase (AMPK) and induction of autophagy. Our results indicated that ETS-1 induced autophagy after inhibition of glycolysis, and thus led to comparative decrease of cell viability. These results implied that ETS-1 could be a potential target for tumor metabolic therapy.

\section{Introduction}

Pancreatic cancer is the fourth leading cause of cancer death and is expected to become the second major cause of cancer mortality in Western countries by 2020 (1). Most cases are diagnosed in the late stage due to the absence of early symptoms and the lack of validated screening tests for early diagnosis, so the overall survival of the patients with pancreatic cancer are limited (2). Current therapeutic strategies, such as surgical resection, radiation and chemotherapy, are unsatisfactory (3-5). Thus, identification and development of more effective treatment strategies is urgently needed.

Tumor cells including pancreatic cancer cells reprogram their metabolism to meet their bioenergetic and biosynthetic requirements, and increased glycolysis is a primary biochemical hallmark of tumors (6). This phenomenon is known as the Warburg effect and is one of the most predominant metabolic alterations that occur during malignant transformation (7). In contrast with normal cells which transform glucose into carbonic anhydride under aerobic conditions, the tumor cells mostly produce lactate, even in the presence of sufficient levels of oxygen (8). It has been documented that tumor glycolysis provides energy for rapid growth and promotes cancer metastasis (9). Thus, tumor growth inhibition of pancreatic cells through targeting and blocking of glycolysis could be an attractive antitumor strategy (4).

V-ets erythroblastosis virus E26 oncogene homolog-1 (ETS-1) belongs to the ETS transcription factor family characterized as a conserved DNA-binding domain (transcription 
activation domain) (10-12). Our previous study (10) and other studies proved the importance of the ETS family members in the regulation of cell development, differentiation, proliferation, apoptosis, angiogenesis, tissue remodeling, migration, invasion, malignant transformation and metastasis (10,13-17). ETS-1 is an important transcription factor regulating MMP genes (18) such as MMP1, MMP3 and MMP9 (18-21). Additionally, it can mediate extracellular matrix degradation, cell migration, angiogenesis and drug resistance $(22,23)$. Considerable attention has been paid to ETS-1 due to its important roles in regulating the energy metabolism in cancer cells $(11,24)$. It has been stated that the overexpression of ETS-1 is responsible for the poor prognosis of breast cancer $(10,25-31)$, ovarian carcinoma (32-34), and cervical carcinoma $(24,35)$. Therefore, ETS-1 may be a promising molecular target for the new therapeutic strategy of pancreatic cancer (36).

Increased uptake of extracellular glucose is facilitated by the glucose transporter-1 (GLUT-1) which is commonly upregulated in a wide range of solid tumors and contribute to the Warburg effect $(37,38)$. It has been shown that GLUT-1 is associated with the development, poor prognosis of cancers and inhibition of glycolysis which can effectively block the proliferation and invasion of the cancer cells. Thus, key transporters that participate in glycolytic metabolism such as GLUT-1 could become vulnerable for pancreatic cancer therapy $(39,40)$.

AMP-activated protein kinase (AMPK) is a heterotrimer comprised of one catalytic subunit $\alpha$ and two regulatory subunits $\beta$ and $\gamma$ and AMPK $\alpha$ phosphorylation site on threonine 172 (pT172) that is essential for full activation of AMPK (41). As a cellular energy sensor, AMPK is activated when the uptaking of glucose is reduced and AMP/ATP ratio is increased (42). AMPK plays a pivotal role in 'sensing' energetic stress. It can promote the catabolism to generate more ATP and inhibit the anabolism of cancer cells to keep a sustained energy supply (43). Furthermore, AMPK has been reported to stimulate the process of autophagy, a cellular process in which components of the cell are degraded to ensure sufficient metabolites in response to nutrient limitation $(44,45)$.

In this study, we sought to detect whether silencing ETS-1 could be a selective anticancer strategy by reducing the expression of GLUT-1 to inhibit glycolysis and then induce autophagy to decrease viability of pancreatic cancer cells.

\section{Materials and methods}

Cell culture and reagents. The two human pancreatic cancer cell lines, Panc-1, PaTu-8988 were cultured in Dulbecco's modified Eagle's medium (DMEM) supplemented with $10 \%$ heat inactivated fetal bovine serum (FBS), $100 \mathrm{U} / \mathrm{ml}$ penicillin and $100 \mu \mathrm{g} / \mathrm{ml}$ streptomycin (Invitrogen, Carlsbad, CA, USA) in a humidified incubator in $5 \% \mathrm{CO}_{2}$ at $37^{\circ} \mathrm{C}$. Cells were in $\log$ phase prior to the following experiments.

Establishing of stable ETS-1 knockdown cell lines. Panc-1 cells and $\mathrm{PaTu}-8988$ cells were seeded in 6 -well plate $\left(5 \times 10^{5}\right.$ cells/well $)$ separately followed by transfection with $500 \mathrm{ng} / \mathrm{ml}$ of either psi-ETS-1 (PIEE102075355) or control plasmids (constructed and analyzed sequence by GeneChem Company, Shanghai, China) conjugated with Lipofectamine 2000 (11668-019;
Table I. Sequences of the oligonucleotide primers.

\begin{tabular}{llc}
\hline Gene & \multicolumn{1}{c}{ Primer sequences $\left(5^{\prime}-3^{\prime}\right)$} & $\begin{array}{c}\text { Product } \\
(\mathrm{bp})\end{array}$ \\
\hline ETS-1 & $\begin{array}{l}\text { F: 5'-GTCGTGGTAAACTCGG-3' } \\
\text { R:5'-CAGCAGGAATGACAGG-3' }\end{array}$ & 246 \\
GLUT-1 & F: 5'-CGGGCCAAGAGTGTGCTAAA-3' & 283 \\
& R:5'-TGACGATACCGGAGCCAATG-3' & \\
ATG5 & F: 5'-AAGCAACTCTGGATGGGATT-3' & 239 \\
& R:5'-GCAGCCACAGGACGAAAC-3' & \\
ATG7 & F: 5'-ACCCAGAAGAAGCTGAACGA-3' & 267 \\
& $\begin{array}{l}\text { R:5'-AGACAGAGGGCAGGATAGCA-3' } \\
\text { GAPDH }\end{array}$ & F: 5'-CCACCCATGGCAAATTCCATGGCA-3' \\
& R:5'-TCTAGACGGCAGGTCAGGTCCACC-3' & \\
\hline
\end{tabular}

ETS-1, v-ets erythroblastosis virus E26 oncogene homolog-1; GLUT-1, glucose transporter-1; ATG5, autophagy-related gene 5; ATG7, autophagy-related gene 7; GAPDH, glyceraldehyde-3-phosphate hydrogenase; F, forward; $\mathrm{R}$, reverse.

Invitrogen). After transfection, cells were cultured in Opti-MEM medium (31985-062; Invitrogen) according to the manufacturer's instructions.

Cell viability assay. Cell viability was assessed by MTT cell proliferation and cytotoxicity assay kit (C0009; Beyotime Biotechnology). Panc-1, PaTu-8988 cells were seeded in 96 -well plates at a density of $\sim 10,000$ cells/well. Thiazolyl blue tetrazolium bromide (MTT) solution was prepared following the instructions, and incubated with the growing cultures at a final concentration of $0.5 \mathrm{mg} / \mathrm{Ml}$ for $4 \mathrm{~h}$ at $37^{\circ} \mathrm{C}$. Absorbance at $570 \mathrm{~nm}$ was read on a multiwell scanning spectrophotometer (Infinite ${ }^{\circledR}$; Tecan, Shanghai China), and the results were expressed as a percentage $(\%)$ of the control.

Reverse transcription-polymerase chain reaction ( $R T-P C R)$. Total RNA was extracted from untreated and stably transfected cells using TRIzol reagent (15596-026; Invitrogen). First-Strand cDNA was synthesized according to the manufacturer's instructions (DRR036A; Takara, Tokyo, Japan). RT-PCR analysis was carried out using 2X Power Taq PCR Master Mix (PR1700; BioTeke, Beijing, China) under the following conditions: $95^{\circ} \mathrm{C}$ for $5 \mathrm{~min}$ to denature cDNA, followed by 30 cycles of $95^{\circ} \mathrm{C}$ for $30 \mathrm{sec}, 55^{\circ} \mathrm{C}$ for $15 \mathrm{sec}$, and $72^{\circ} \mathrm{C}$ for $30 \mathrm{sec}$, followed by a terminal extension for $7 \mathrm{~min}$ at $72^{\circ} \mathrm{C}$. PCR products were electrophoresed on a $2 \%$ agarose gel containing ethidium bromide (16550-100; Invitrogen) at $90 \mathrm{~V}$ for $45 \mathrm{~min}$, and the bands were visualized using the UltraPower $^{\mathrm{TM}}$ Gel Imaging system (EP2018; BioTeke). The primer sequences are listed in Table I. The primers were obtained from Invitrogen.

Glucose uptake and lactate release assay. The culture contains $4.5 \mathrm{~g} / 1$ glucose which will be uptaken by cancer cells to supply energy and produce lactate. The supernatants from untreated and stably transfected cells of Panc-1, PaTu-8988 cultures were collected respectively at various time points. Glucose 
uptake rate in culture supernatants was determined using a glucose assay kit (F006; Nanjing Jiancheng Bioengineering Institute, Nanjing, China) according to the manufacturer's instructions, and quantified by absorption at $450 \mathrm{~nm}$. Lactate generation was measured using a lactate assay kit according to the manufacturer's instruction (A019-2; Nanjing Jiancheng Bioengineering Institute).

ATP detection by colorimetric measurements. Intracellular ATP was measured by a luciferin-luciferase method using ATP assay kit (A095; Nanjing Jiancheng Bioengineering Institute). Briefly, untreated and stably transfected cells of Panc-1, PaTu-8988 were collected after washing with PBS. Boiling distilled water was added to the collected cells and then the suspension was placed in hot bath to homogenize the cells. After that the suspension was heated in boiling water bath for $10 \mathrm{~min}$ and eddied for $1 \mathrm{~min}$. The supernatants were collected after centrifugation $\left(12,000 \mathrm{x} \mathrm{g}, 4^{\circ} \mathrm{C}\right)$ for $10 \mathrm{~min}$ (Eppendorf Centrifuge 5424R), followed by mixing with the ATP detection buffer for analyzing by luminescence spectrometry. The final ATP content of each sample was normalized to protein concentration measured by BCA Protein assay kit (A045-2; Nanjing Jiancheng Bioengineering Institute).

Western blot analyses. Total proteins of cultured cells were extracted using RIPA buffer containing phenylmethanesulfonyl fluoride (PMSF). BCA protein assay kit (G2026; Wuhan Guge Bioengineering Institute, Wuhan, China) was used to determine the protein concentrations. Samples were resolved by $12.5 \%$ SDS-PAGE (Invitrogen) after mixing with SDS-PAGE loading buffer and boiling for 5 min. Proteins were transferred to a PVDF membranes and diluted in blocking buffer (5\% dry milk) for $1 \mathrm{~h}$, and then incubated separately with diluted (1:300) primary antibodies [polyclonal rabbit anti-ETS-1, GLUT-1, autophagy-related gene 5 (ATG5), ATG7, AMPK, light chain 3 (LC3); Wuhan Guge Bioengineering Institute] overnight. Then, secondary HRP-conjugated antibody (1:3,000, GB23301; Wuhan Guge Bioengineering Institute) was added for $1 \mathrm{~h}$. Membrane was exposed using the chemiluminescence detection kit (G2019; Wuhan Guge Bioengineering Institute). Anti- $\beta$-actin antibody (GB13001; Wuhan Guge Bioengineering Institute) was used as control.

Statistical analysis. GraphPad Prism 5.0 (GraphPad Software, San Diego, CA, USA) was used for all statistical analysis. The mean $\pm \mathrm{SD}$ was determined for each group in the individual experiments. The Student's t-test was used to determine the significance of differences between different groups. P-values $<0.05$ were statistically significant.

\section{Results}

Transcriptional silencing of ETS-1 attenuated cell viability in Panc-1 and PaTu-8988 cells. To evaluate the function of ETS-1 gene in the regulation of GLUT-1 expression in pancreatic cancer cells, two stable ETS-1 silencing cell lines, Panc-1-siETS-1 and PaTu-8988-siETS-1, were established using a green fluorescent protein (GFP)-expressing adenoviral vector carrying an shRNA targeting the ETS-1 gene.
The success of the plasmid transfection was monitored by brightfield and fluorescence images (magnification, x100). Our results showed the plasmid was successfully transfected in Panc-1 and PaTu-8988 cells (Fig. 1A). The silencing efficiency of the ETS-1 shRNA was determined using qRT-PCR and western blot analysis. qRT-PCR results showed that mRNA expression level of ETS-1 in Panc-1-siETS-1 and PaTu-8988-siETS-1 transfected cells was reduced with a percentage of 68 and $52 \%$, respectively compared with control cells (Fig. 1B). Additionally, western blot analysis indicated the level of ETS-1 protein was also significantly decreased in the ETS-1-silenced cells with a percentage of 88.50 and 80.44\% for Panc-1-siETS-1 and PaTu-8988-siETS-1 cells, respectively (Fig. 1C).

In order to assess the cell viability of pancreatic cancer cells, MTT assay was performed, the result demonstrated that the cell viability was significantly reduced in Panc-1 and PaTu-8988 cells with a percentage of 200 and $180 \%$ after transfection for $72 \mathrm{~h}$, respectively (Fig. 2). Collectively these results indicated that silencing ETS-1 by transfecting adenoviral vectors carrying shRNA targeting the ETS-1 gene in pancreatic cancer cells could reduce cell viability.

ETS-1 knockdown inhibits glucose metabolism and activated AMPK. Pancreatic cancer cells predominantly metabolize glucose transported by GLUT-1 to lactate to meet energy demand. In order to detect the mechanism of inhibition the glucose metabolism after silencing ETS-1 in pancreatic cancer cells, we measured glucose uptake rate, lactate release rate by standard colorimetric assay kits in Panc-1, PaTu-8988, Panc-1-siETS-1 and PaTu-8988-siETS-1 silenced cells. We found that glucose uptake rate was reduced in Panc-1-siETS-1 and PaTu-8988-siETS-1 cells with 5.36 and $4.17 \%$, respectively compared with control group (Fig. 3A). Similarly, lactate releasing rate was also reduced in Panc-1-siETS-1 and PaTu-8988-siETS-1 cells with 131.7 and $142.69 \%$, respectively compared with control group (Fig. 3B).

GLUT-1 is a predominant glucose transporter in human cells. Previous studies have documented that upregulation of GLUT-1 plays an essential role in cancer cells proliferation (46). To measure the GLUT-1 expression level in ETS-1 silenced cells, qRT-PCR and western blot analysis were performed. The results revealed that silencing ETS-1 inhibited the mRNA level of glycolysis-related GLUT-1 expression with a ratio of $67 \%$ in Panc-1-siETS-1 cells and 29\% in PaTu-8988-siETS-1 cells compared with control group (Fig. 3C). Interestingly, the GLUT-1 protein level was also reduced with $93.65 \%$ in Panc-1-siETS-1 cells and $66.20 \%$ in PaTu-8988-siETS-1 cells compared with control cells (Fig. 3D).

Cancer cells could utilize glucose that was mainly transported by GLUT-1 through glycolysis to produce lactate and yield energy in the form of ATP. The decreased production of ATP could stimulate the process of AMPK phosphorylation. To measure cellular ATP level, standard colorimetric assay kits were performed in pancreatic cancer cells. The results showed that cellular ATP formation was decreased from $10.32 \mu \mathrm{mol} / \mathrm{g}$ protein to $8.28 \mu \mathrm{mol} / \mathrm{g}$ protein and from $12.16 \mu \mathrm{mol} / \mathrm{g}$ protein to $8.43 \mu \mathrm{mol} / \mathrm{g}$ protein in Panc-1-siETS-1 and PaTu-8988-siETS-1 
A Panc-1

shNC
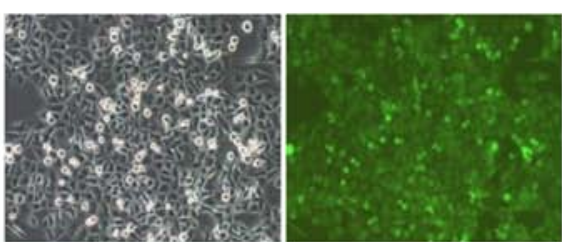

ShRNA

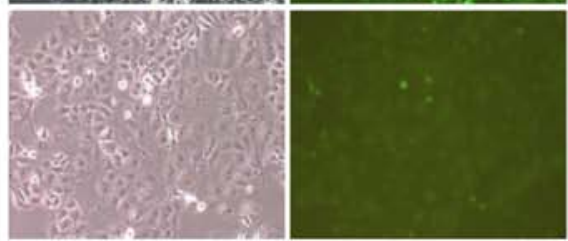

B

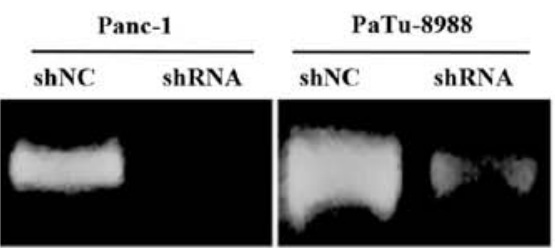

GAPDH

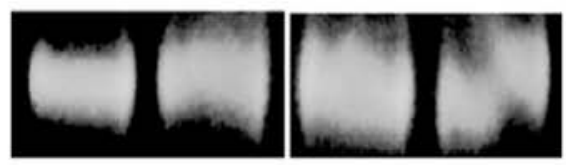

C

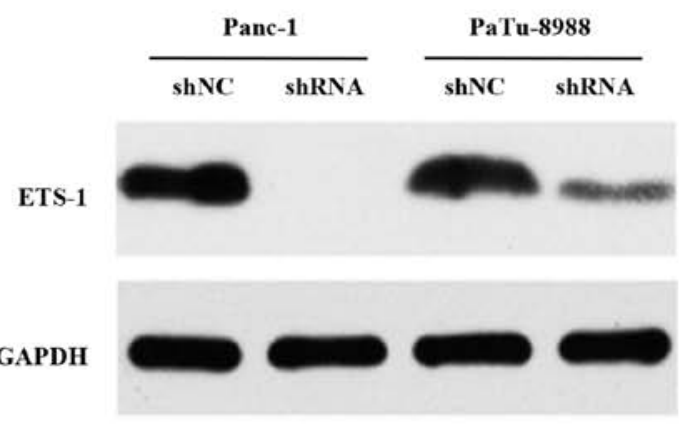

PaTu-8988

$\operatorname{shNC}$
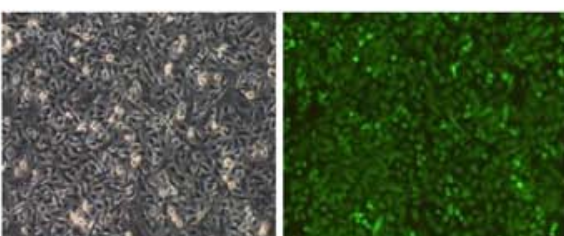

ShRNA
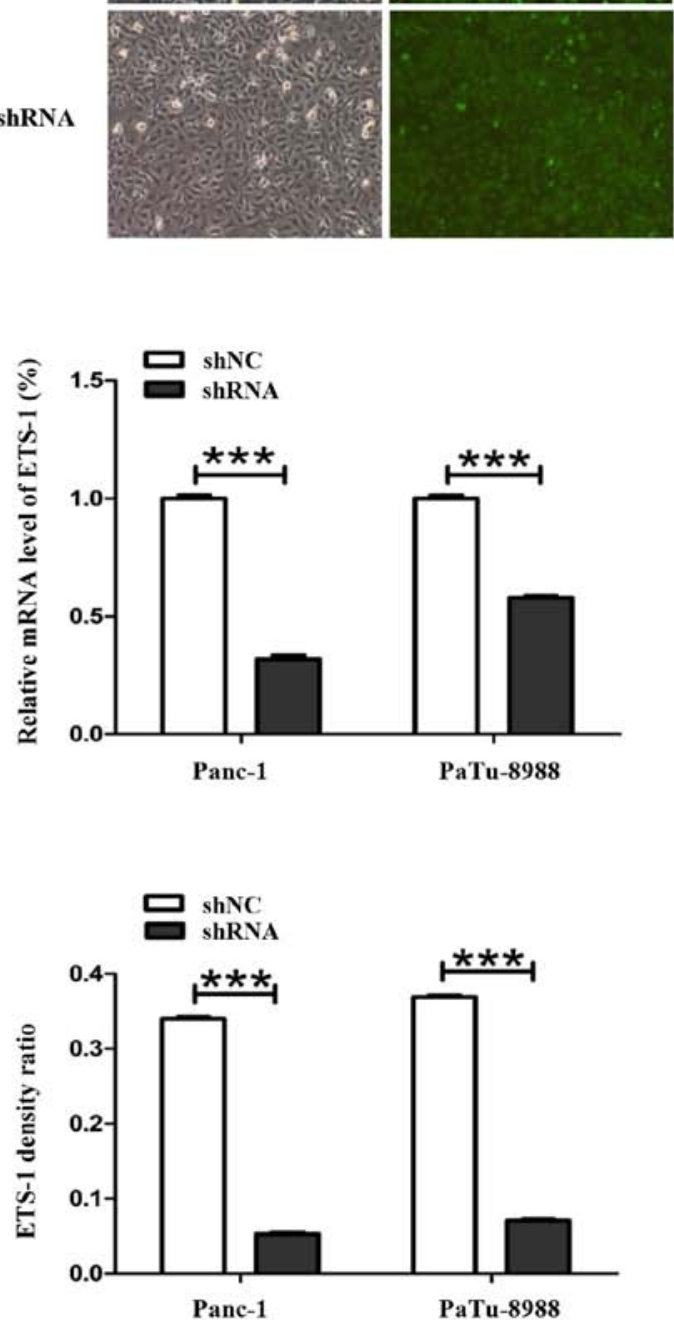

Figure 1. Efficiency of ETS-1 transcriptional silencing. Expression level of ETS-1 in Panc-1 and PaTu-8988 cells was decreased after ETS-1 shRNA transfection. (A) Brightfield and fluorescence images (magnification, x100).(B) ETS-1 mRNA expression levels in ETS-1 shRNA and control shNC-transfected cells were analyzed by qRT-PCR and quantified by ImageJ. Data are presented as mean \pm SD of three independent experiments, ${ }^{* * *} \mathrm{P}<0.001$. (C) Western blot analysis of protein expression of ETS-1 in ETS-1 shRNA silenced cell lines and control shNC cells. Band densities in western blot analysis were quantified by ImageJ. GAPDH was included as a normalization control. ETS-1, v-ets erythroblastosis virus E26 oncogene homolog-1; GAPDH, glyceraldehyde 3-phosphate dehydrogenase.
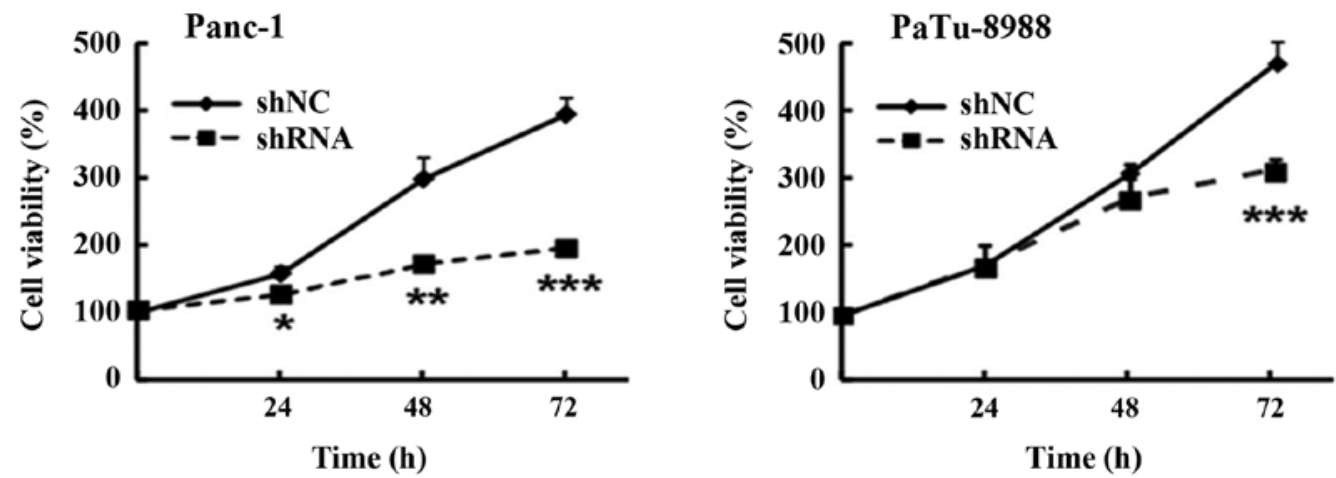

Figure 2. Pancreatic cancer cell viability is inhibited by silencing ETS-1. Cell viability (OD value) of Panc-1, PaTu-8988, Panc-1-siETS-1 and PaTu-8988-siETS-1 was assessed by MTT. Data are presented as mean \pm SD from three independent experiments, ${ }^{*} \mathrm{P}<0.05,{ }^{* * *} \mathrm{P}<0.01$ and ${ }^{* * * *} \mathrm{P}<0.001$ 
A

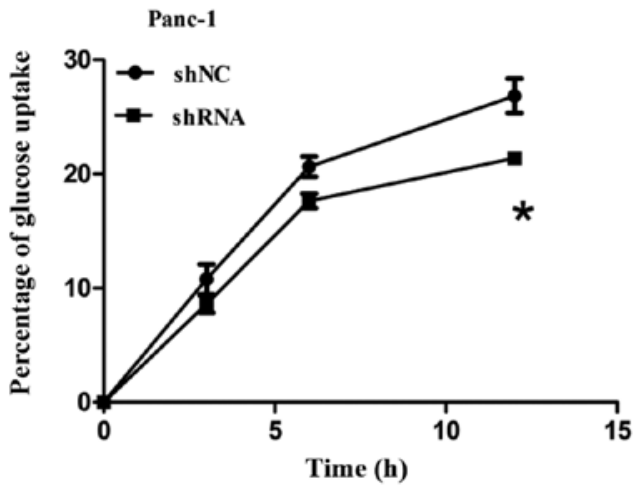

B

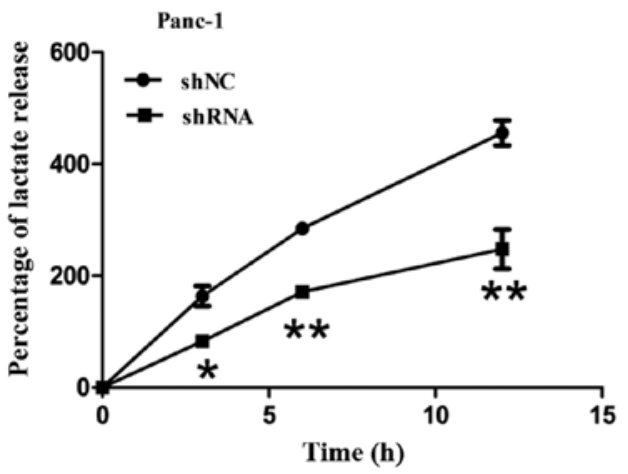

C

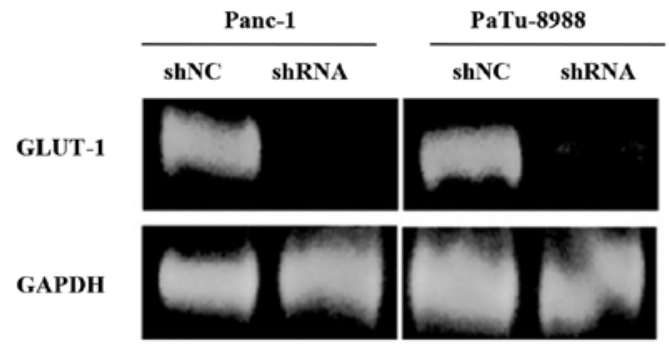

D

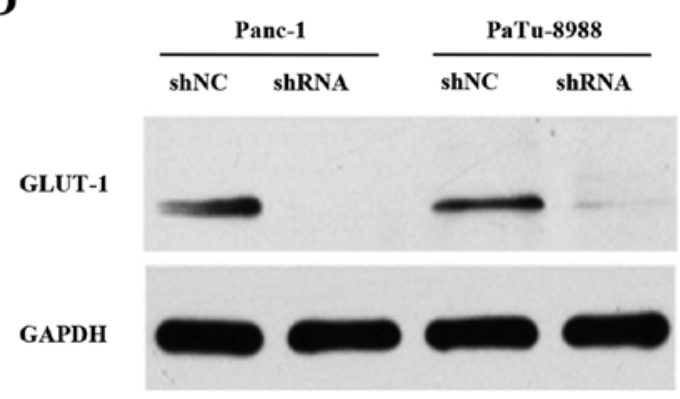

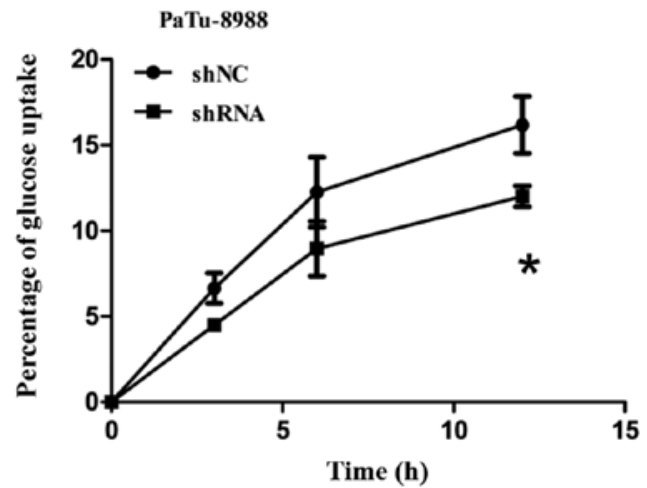
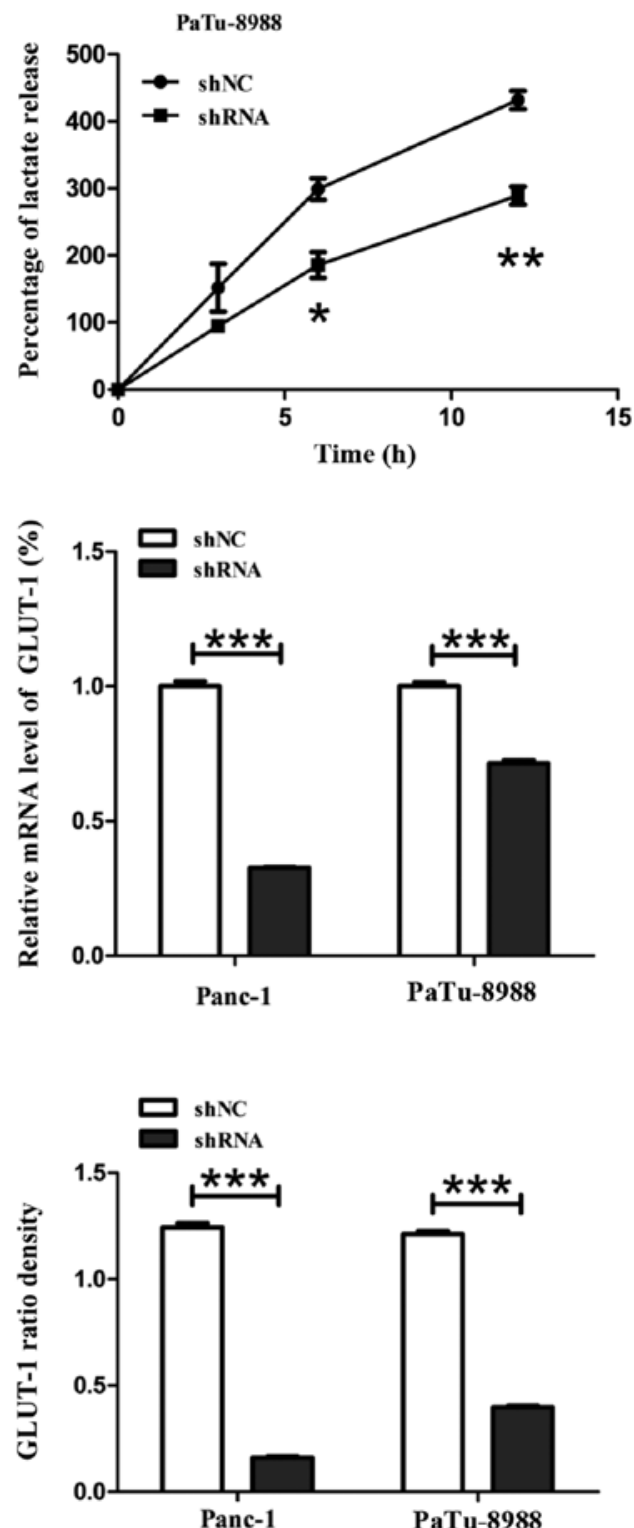

Figure 3. Glucose consumption, lactate production and GLUT-1 expression was decreased after silencing ETS-1. The concentration of glucose and lactate in supernatant were measured by standard colorimetric assay kits at 4,8 and $12 \mathrm{~h}$. (A) Glucose absorption rate was measured in Panc-1-siETS-1, PaTu-8988-siETS-1 cells and their control cells. (B) Lactate release rate of silencing ETS-1 cells and control cells. (C) qRT-PCR analysis and ImageJ quantification of mRNA expression levels of GLUT-1 in Panc-1, PaTu-8988, Panc-1-siETS-1 and PaTu-8988-siETS-1 cells. (D) GLUT-1 protein levels in ETS-1-silenced cell lines and control cells were analyzed by western blot analysis and quantified by ImageJ. Data are presented as mean $\pm \mathrm{SD}$ of three independent experiments, ${ }^{*} \mathrm{P}<0.05$, ${ }^{* *} \mathrm{P}<0.01$ and ${ }^{* * *} \mathrm{P}<0.001$. GLUT-1, glucose transporter 1 .

cells, respectively, compared to control cells (Fig. 4A). Besides, the AMPK phosphorylation was increased 2.36- and 1.29-fold in Panc-1-siETS-1 and PaTu-8988-siETS-1 cells compared with control cells (Fig. 4B). 
A

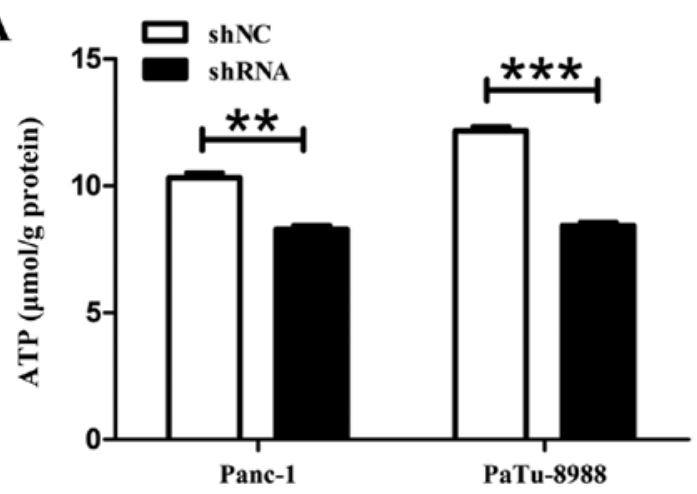

B
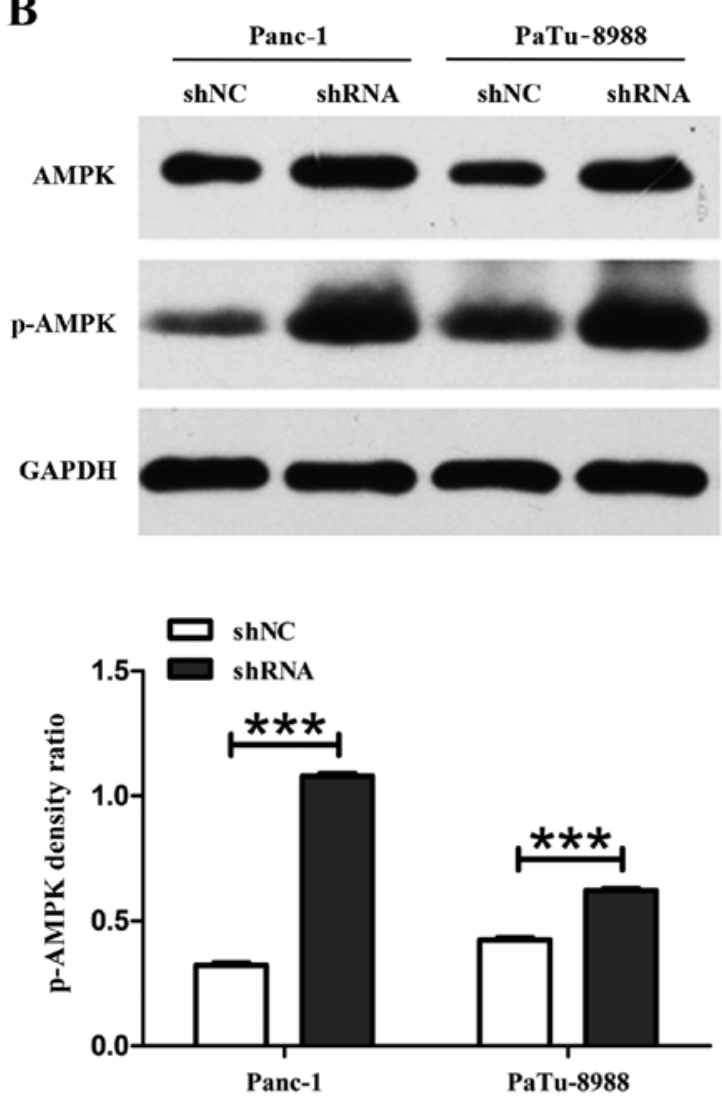

Figure 4. The ATP production and expression of p-AMPK protein in pancreatic cancer cells. (A) The ATP levels were detected by standard colorimetric assay kits in Panc-1, Panc-1-siETS-1, PaTu-8988, PaTu-8988-siETS-1 cells (B) Western blot analysis of p-AMPK protein in ETS-1 silencing cells and control groups. Band densities in western blot analysis were quantified by ImageJ. Data are presented as mean \pm SD of three independent experiments, ${ }^{* *} \mathrm{P}<0.01$ and ${ }^{* * *} \mathrm{P}<0.001$

These findings indicated that glucose metabolism was inhibited through decreasing the level of GLUT-1 expression after ETS-1 knockdown followed by AMPK activation.

Transcriptional silencing of ETS-1 induces autophagy. Autophagy is an intracellular lysosomal degradation processs (47) by which cells capture intracellular proteins, lipids and organelles, and deliver them to the lysosomal compartment for degradation. It has been documented that autophagy promotes cell survival upon nutrient depletion such as glucose starvation. Among different kinds of autophagy-related (Atg) proteins, ATG5 and ATG7 proteins play an essential role in the formation of preautophagosome and isolation membrane $(48,49)$.

In the previous sections, we demonstrated that ETS-1 interference can attenuate the cell viability and inhibit glycolysis. In order to investigate the mechanism of the attenuation on cell viability after silencing ETS-1, qRT-PCR and western blot analysis were used to analyze the expression level of autophagy-related genes in Panc-1-siETS-1, PaTu-8988-siETS-1, Panc-1 and PaTu-8988 cells. qRT-PCR data showed that the mRNA level of ATG5 was increased 3.34- and 3.57-fold in Panc-1-siETS-1 and PaTu-8988-siETS-1 cells, respectively, compared with control cells. Similarly, the mRNA level of ATG7 was also increased 1.75- and 3.84-fold in Panc-1-siETS-1 cells and PaTu-8988-siETS-1 cells, respectively, compared with control group (Fig. 5A). Furthermore, the ATG5 protein expression was increased 1.58- and 1.64-fold in Panc-1-siETS-1 and PaTu-8988T-siETS-1 cells, respectively, compared with control cells. The protein expression of ATG7 was also increased 2.67-fold in Panc-1-siETS-1 cells and 1.89-fold in PaTu-8988-siETS-1 cells compared with control cells (Fig. 5B).

Microtubule-associated protein LC3 is a mammalian homolog gene of ATG8 in yeast. LC3I combined with PE to form LC3II, which is a marker located on the membrane of the autophagosome during the induction of autophagy (48).

In order to verify whether the attenuation of cell viability was caused by autophagy, we further analyzed the protein expression of LC3II by western blot assay. The results showed that the protein level of LC3II was increased 3.37- and 1.54-fold, respectively, compared with internal marker in Panc-1-siETS-1 and PaTu-8988T-siETS-1 cells (Fig. 5C).

Collectively, these results indicated that silencing of ETS-1 can attenuate the cell viability of pancreatic cancer by decreasing the expression of GLUT-1. The silencing of ETS-1 can obviously decrease the expression of GLUT-1 and subsequently interfere with glycolysis and induce autophagy.

\section{Discussion}

Pancreatic cancer remains one of the most lethal malignancies due to its aggressiveness and its intrinsic resistance to standard chemotherapy. This is possibly due to a low vascular density and a prominent fibrotic stromal compartment impairing successful drug delivery to cancer cells. Therefore, new effective treatments are urgently needed. Previous studies have noted that pancreatic cancer cells, even under non-hypoxic conditions, predominantly employ cytosolic glycolysis and lactate fermentation rather than mitochondrial oxidative phosphorylation of pyruvate for their energy production. This metabolic pathway is called 'Warburg effect', and was first described in the 1920s by Otto Warburg (37). Thus, we concluded that targeting the metabolic reprogramming in cancers could be a promising strategy for pancreatic cancer treatment.

ETS-1, a member of ETS transcription factor family, playing a vital role in regulating differentiation, proliferation, apoptosis, angiogenesis, migration, metastasis and metabolism of cancer cells. Our previous studies have demonstrated that high expression of transcription factor ETS-1 is associated with migration, invasion and metastasis of cancer cells, thus 

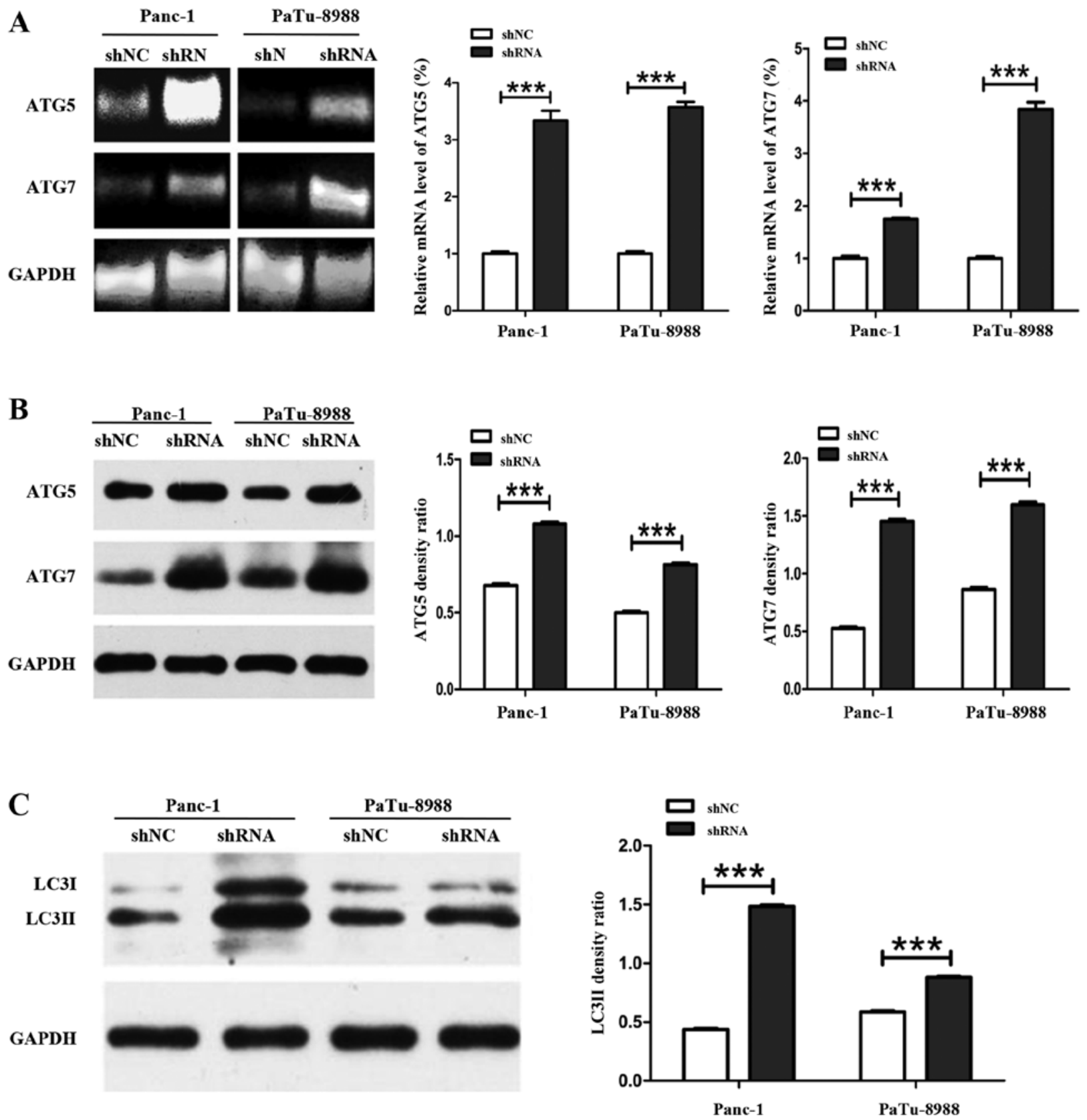

Figure 5. Silencing ETS-1 induces autophagy in pancreatic cancer cells. (A) qRT-PCR analysis and ImageJ quantification of ATG-5 and ATG-7 mRNA expression levels in ETS-1-silenced cells and control groups. (B) ATG-5 and ATG-7 protein levels were analyzed by western blot analysis and quantified by ImageJ in ETS-1-silenced cells and control groups. (C) Western blot analysis and ImageJ quantification of LC3II in ETS-1-silenced cells and control cells. Data are presented as mean $\pm \mathrm{SD}$ of three independent experiments, ${ }^{* * * *} \mathrm{P}<0.001$.

ETS-1 could be used as a prognostic biomarker in pancreatic cancer (10), we also found that overexpression of ETS-1 enhanced the dependence on glycolysis for cellular energy production in pancreatic cancer cells. Metabolic alterations including the increasing of glycolysis dependence are considered as common characteristic of cancer cells to rapidly generate energy, support the rapid cell division, uncontrolled proliferation, and distant metastasis of tumor cells (50). In this study, we sought to identify the potential role for ETS-1 in the regulation of glucose metabolism and evaluate whether it could be a novel target for metabolic therapy in pancreatic cancer. First and foremost, our results showed that transcriptional silencing of ETS-1 attenuated cell viability in Panc-1 and PaTu-8988 cells. This finding is consistent with previous studies that have demonstrated blocking major pathways such as glycolysis may be successful in reducing cell growth or tumorigenicity. Therefore, it could be concluded that inhibiting the reprogramming metabolism pathways could provide a new chance for tumor treatment.

GLUT-1 is involved in aerobic glycolysis (Warburg effect) via facilitating glucose transport to generate energy $(51,52)$. In this study, our results showed that si-RNA mediated knockdown of ETS-1 obviously downregulating the level of GLUT-1. We also found that inhibition of GLUT-1 expression significantly reduced the level of glucose uptake, lactate secretion and intracellular ATP production. The decrease in ATP generation level is associated with the activation of AMPK via phosphorylation relying on liver kinase B1 (LKB1) processing (41). AMPK is a 
hetero-trimeric protein complex that plays an essential role in the regulation of metabolic and energy homeostasis in cancer cells. Under nutrient-abundant condition, AMPK is suppressed and mTOR is activated, by which promotes generation of biomass that leads to cell growth and proliferation. While under glucose-limiting condition, AMPK, by activating p38 and by inhibiting mTOR, regulates expression of PGC1- $\alpha$, which controls mitochondrial biogenesis in cancer cells, thereby allowing oxidative metabolism of non-glucose carbon sources, such as glutamine, lactate and fatty acids, to generate ATP. It is important to mention that autophagy is promoted by AMPK via suppressing mTORC1 and activating ULK1. Autophagy, an alternative metabolic pathway, is an important catabolic program to recycle the energy and nutrients to sustain the survival of cancer cells under nutrient and metabolic stress (53). The autophagy is utilized as a cytoprotective mechanism to rescue cells in energy-deprived condition, while this kind of insufficient activation of autophagy may also improve the sensation of chemotherapeutics and ionizing radiation in tumor cells (48). In addition, autophagy has been reported as a major mechanism to induce cancer cell death called type II programmed cell death in the absence of apoptosis which is blocked or defective in certain tumor cells (54). In this study we found that LC3II, the marker of autophagy, increased in si-ETS-1 cells compared with control groups. Importantly we found that autophagy-related proteins ATG5/7, was obviously increased at mRNA and protein levels. These results indicated that ETS-1 can reduce the expression of GLUT-1 to interfere with the energy production of cancer cells and activate AMPK to prompt autophagy.

These findings suggest that the silence of ETS-1 in pancreatic cancer cells disturb the expression of GLUT-1 to reduce the absorption of glucose and production of lactate to impair the glycolysis through alteration of 'Warburg effect', resulting in AMPK activation, autophagy induction and reduction of cell viability.

An important implication of these findings is that ETS-1 plays functionally significant roles in attenuating pancreatic cancer cell viability by decreasing the expression of GLUT-1 to interfere glycolysis and induce autophagy. In our future research, we aim to combine the strategy of inhibiting glycolysis with different modulators of autophagy and detect their efficacy in clinical application.

\section{Acknowledgements}

This study was supported financially by the National Nature Science Foundation of People's Republic of China (81271699).

\section{References}

1. Perera RM and Bardeesy N: Pancreatic cancer metabolism: Breaking it down to build it back up. Cancer Discov 5: 1247-1261, 2015.

2. Xie D and Xie K: Pancreatic cancer stromal biology and therapy. Genes Dis 2: 133-143, 2015.

3. Cid-Arregui A and Juarez V: Perspectives in the treatment of pancreatic adenocarcinoma. World J Gastroenterol 21: 9297-9316, 2015.

4. Guillaumond F, Iovanna JL and Vasseur S: Pancreatic tumor cell metabolism: Focus on glycolysis and its connected metabolic pathways. Arch Biochem Biophys 545: 69-73, 2014.
5. Paniccia A, Merkow J, Edil BH and Zhu Y: Immunotherapy for pancreatic ductal adenocarcinoma: An overview of clinical trials. Chin J Cancer Res 27: 376-391, 2015.

6. Tennant DA, Durán RV and Gottlieb E: Targeting metabolic transformation for cancer therapy. Nat Rev Cancer 10: 267-277, 2010.

7. Lee M and Yoon JH: Metabolic interplay between glycolysis and mitochondrial oxidation: The reverse Warburg effect and its therapeutic implication. World J Biol Chem 6: 148-161, 2015.

8. Zhou W, Capello M, Fredolini C, Piemonti L, Liotta LA, Novelli F and Petricoin EF: Proteomic analysis of pancreatic ductal adenocarcinoma cells reveals metabolic alterations. J Proteome Res 10: 1944-1952, 2011.

9. Lunt SY and Vander Heiden MG: Aerobic glycolysis: Meeting the metabolic requirements of cell proliferation. Annu Rev Cell Dev Biol 27: 441-464, 2011.

10. Li C, Wang Z, Chen Y, Zhou M, Zhang H, Chen R, Shi F, Wang $C$ and Rui Z: Transcriptional silencing of ETS-1 abrogates epithelial-mesenchymal transition resulting in reduced motility of pancreatic cancer cells. Oncol Rep 33: 559-565, 2015.

11. Verschoor ML, Verschoor CP and Singh G: Ets-1 global gene expression profile reveals associations with metabolism and oxidative stress in ovarian and breast cancers. Cancer Metab 1: 17, 2013.

12. Zhu M, Li M, Zhang F, Feng F, Chen W, Yang Y, Cui J, Zhang D and Linghu E: FBI-1 enhances ETS-1 signaling activity and promotes proliferation of human colorectal carcinoma cells. PLoS One 9: e98041, 2014.

13. Shaikhibrahim Z and Wernert N: ETS transcription factors and prostate cancer: The role of the family prototype ETS-1 (Review). Int J Oncol 40: 1748-1754, 2012.

14. Lelièvre E, Lionneton F, Soncin F and Vandenbunder B: The Ets family contains transcriptional activators and repressors involved in angiogenesis. Int J Biochem Cell Biol 33: 391-407, 2001.

15. Oikawa T: ETS transcription factors: Possible targets for cancer therapy. Cancer Sci 95: 626-633, 2004.

16. Hahne JC, Okuducu AF, Kaminski A, Florin A, Soncin F and Wernert N: Ets-1 expression promotes epithelial cell transformation by inducing migration, invasion and anchorageindependent growth. Oncogene 24: 5384-5388, 2005.

17. Shaikhibrahim Z, Langer B, Lindstrot A, Florin A, Bosserhoff A, Buettner R and Wernert N: Ets-1 is implicated in the regulation of androgen co-regulator FHL2 and reveals specificity for migration, but not invasion, of PC3 prostate cancer cells. Oncol Rep 25: 1125-1129, 2011.

18. Gao H, Peng C, Liang B, Shahbaz M, Liu S, Wang B, Sun Q, Niu Z, Niu W, Liu E, et al: $\beta 6$ integrin induces the expression of metalloproteinase-3 and metalloproteinase-9 in colon cancer cells via ERK-ETS1 pathway. Cancer Lett 354: 427-437, 2014.

19. Bu S, Yamanaka M, Pei H, Bielawska A, Bielawski J, Hannun YA Obeid L and Trojanowska M: Dihydrosphingosine 1-phosphate stimulates MMP1 gene expression via activation of ERK1/2-Ets1 pathway in human fibroblasts. FASEB J 20: 184-186, 2006.

20. Li T and Jiang S: Effect of bFGF on invasion of ovarian cancer cells through the regulation of Ets-1 and urokinase-type plasminogen activator. Pharm Biol 48: 161-165, 2010.

21. Jiang Y, Xu W, Lu J, He F and Yang X: Invasiveness of hepatocellular carcinoma cell lines: Contribution of hepatocyte growth factor, c-met, and transcription factor Ets-1. Biochem Biophys Res Commun 286: 1123-1130, 2001.

22. Tomita N, Morishita R, Taniyama Y, Koike H, Aoki M, Shimizu H, Matsumoto K, Nakamura T, Kaneda Y and Ogihara T: Angiogenic property of hepatocyte growth factor is dependent on upregulation of essential transcription factor for angiogenesis, ets-1. Circulation 107: 1411-1417, 2003

23. Kato T, Fujita Y, Nakane K, Kojima T, Nozawa Y, Deguchi T and Ito M: ETS1 promotes chemoresistance and invasion of paclitaxel-resistant, hormone-refractory PC3 prostate cancer cells by upregulating MDR1 and MMP9 expression. Biochem Biophys Res Commun 417: 966-971, 2012.

24. Verschoor ML, Wilson LA, Verschoor CP and Singh G: Ets-1 regulates energy metabolism in cancer cells. PLoS One 5: e13565, 2010.

25. Sato T and Miwa A: Ets-1 and integrin beta3 for lung metastasis from colorectal cancer. APMIS 110: 347-353, 2002.

26. Sasaki H, Yukiue H, Moiriyama S, Kobayashi Y, Nakashima Y, Kaji M, Kiriyama M, Fukai I, Yamakawa Y and Fujii Y: Clinical significance of matrix metalloproteinase-7 and Ets-1 gene expression in patients with lung cancer. J Surg Res 101: 242-247, 2001. 
27. Verschoor ML and Singh G: Ets-1 regulates intracellular glutathione levels: Key target for resistant ovarian cancer. Mol Cancer 12: 138, 2013.

28. Li W, Zang W, Liu P, Wang Y, Du Y, Chen X, Deng M, Sun W, Wang L, Zhao G, et al: MicroRNA-124 inhibits cellular proliferation and invasion by targeting Ets-1 in breast cancer. Tumour Biol 35: 10897-10904, 2014.

29. Tang N, Wang X, Huang T, Wu Y and Chen Y: Role of Ets-1 in fibronectin-derived heparin-binding domain polypeptides alleviating melanoma cell invasiveness and chemoresistance. Exp Dermatol 23: 512-513, 2014.

30. Span PN, Manders P, Heuvel JJ, Thomas CM, Bosch RR, Beex LV and Sweep CG: Expression of the transcription factor Ets-1 is an independent prognostic marker for relapse-free survival in breast cancer. Oncogene 21: 8506-8509, 2002.

31. Puzovic V, Brcic I, Ranogajec I and Jakic-Razumovic J: Prognostic values of ETS-1, MMP-2 and MMP-9 expression and co-expression in breast cancer patients. Neoplasma 61: 439-446, 2014.

32. Davidson B, Reich R, Goldberg I, Gotlieb WH, Kopolovic J, Berner A, Ben-Baruch G, Bryne M and Nesland JM: Ets-1 messenger RNA expression is a novel marker of poor survival in ovarian carcinoma. Clin Cancer Res 7: 551-557, 2001.

33. Davidson B, Risberg B, Goldberg I, Nesland JM, Berner A, Tropé CG, Kristensen GB, Bryne M and Reich R: Ets-1 mRNA expression in effusions of serous ovarian carcinoma patients is a marker of poor outcome. Am J Surg Pathol 25: 1493-1500, 2001.

34. Takai N, Miyazaki T, Nishida M, Nasu K and Miyakawa I: c-Ets1 is a promising marker in epithelial ovarian cancer. Int $\mathrm{J}$ Mol Med 9: 287-292, 2002.

35. Fujimoto J, Aoki I, Toyoki H, Khatun S and Tamaya T: Clinical implications of expression of ETS-1 related to angiogenesis in uterine cervical cancers. Ann Oncol 13: 1598-1604, 2002.

36. Kimmelman AC: Metabolic dependencies in RAS-driven cancers. Clin Cancer Res 21: 1828-1834, 2015.

37. Cairns RA: Drivers of the Warburg phenotype. Cancer J 21: 56-61, 2015.

38. Mogi A, Koga K, Aoki M, Hamasaki M, Uesugi N, Iwasaki A Shirakusa T, Tamura K and Nabeshima K: Expression and role of GLUT-1, MCT-1, and MCT-4 in malignant pleural mesothelioma. Virchows Arch 462: 83-93, 2013.

39. Blum R and Kloog Y: Metabolism addiction in pancreatic cancer. Cell Death Dis 5: e1065, 2014.

40. Granja S, Pinheiro C, Reis RM, Martinho O and Baltazar F: Glucose addiction in cancer therapy: Advances and drawbacks. Curr Drug Metab 16: 221-242, 2015.

41. Li N, Huang D, Lu N and Luo L: Role of the LKB1/AMPK pathway in tumor invasion and metastasis of cancer cells (Review). Oncol Rep 34: 2821-2826, 2015.
42. Kim N, Jeong S, Jing K, Shin S, Kim S, Heo JY, Kweon GR, Park SK, Wu T, Park JI, et al: Docosahexaenoic acid induces cell death in human non-small cell lung cancer cells by repressing mTOR via AMPK activation and PI3K/Akt inhibition. BioMed Res Int 2015: 239764, 2015.

43. Saito Y, Chapple RH, Lin A, Kitano A and Nakada D: AMPK protects leukemia-initiating cells in myeloid leukemias from metabolic stress in the bone marrow. Cell Stem Cell 17: 585-596, 2015.

44. Mihaylova MM and Shaw RJ: The AMPK signalling pathway coordinates cell growth, autophagy and metabolism. Nat Cell Biol 13: 1016-1023, 2011.

45. Kim J, Kundu M, Viollet B and Guan KL: AMPK and mTOR regulate autophagy through direct phosphorylation of Ulk1. Nat Cell Biol 13: 132-141, 2011.

46. Abdou AG, Eldien MM and Elsakka D: GLUT-1 Expression in cutaneous basal and squamous cell carcinomas. Int J Surg Pathol 23: 447-453, 2015

47. Vuppalapati KK, Bouderlique T, Newton PT, Kaminskyy VO, Wehtje H, Ohlsson C, Zhivotovsky B and Chagin AS: Targeted deletion of autophagy genes Atg5 or Atg7 in the chondrocytes promotes caspase-dependent cell death and leads to mild growth retardation. J Bone Miner Res 30: 2249-2261, 2015.

48. Lao $\mathrm{Y}$ and $\mathrm{Xu} \mathrm{N}$ : Autophagy in cancer chemoprevention: Identification of novel autophagy modulators with anticancer potential. Methods Mol Biol 1379: 151-163, 2016.

49. Shimizu S, Arakawa S and Nishida Y: Autophagy takes an alternative pathway. Autophagy 6: 290-291, 2010.

50. Cairns RA, Harris IS and Mak TW: Regulation of cancer cell metabolism. Nat Rev Cancer 11: 85-95, 2011.

51. Zhang TB, Zhao Y, Tong ZX and Guan YF: Inhibition of glucosetransporter 1 (GLUT-1) expression reversed Warburg effect in gastric cancer cell MKN45. Int J Clin Exp Med 8: 2423-2428, 2015.

52. Yoon SO, Jeon TJ, Park JS, Ryu YH, Lee JH, Yoo JS, Kim JK, Yoon DS and Oh EJ: Analysis of the roles of glucose transporter 1 and hexokinase 2 in the metabolism of glucose by extrahepatic bile duct cancer cells. Clin Nucl Med 40: e178-e182, 2015.

53. Kim SE, Park HJ, Jeong HK, Kim MJ, Kim M, Bae ON and Baek SH: Autophagy sustains the survival of human pancreatic cancer PANC-1 cells under extreme nutrient deprivation conditions. Biochem Biophys Res Commun 463: 205-210, 2015.

54. Liu B, Cheng Y, Liu Q, Bao JK and Yang JM: Autophagic pathways as new targets for cancer drug development. Acta Pharmacol Sin 31: 1154-1164, 2010. 